\title{
Comparison of Measured Dark Current Distributions with Calculated Damage Energy Distributions in HgCdTe
}

\author{
C. J. Marshall, P. W. Marshall, Member, IEEE, C. L. Howe, Student Member, IEEE, R.A. Reed, \\ Member, IEEE, R. A. Weller, Senior Member, IEEE, M. Mendenhall, A. Waczynski, R. Ladbury, \\ and T. M. Jordan
}

\begin{abstract}
This paper presents a combined Monte Carlo and analytic approach to the calculation of the pixel-to-pixel distribution of proton-induced damage in a $\mathrm{HgCdTe}$ sensor array and compares the results to measured dark current distributions after damage by $63 \mathrm{MeV}$ protons. The moments of the Coulombic, nuclear elastic and nuclear inelastic damage distributions were extracted from Monte Carlo simulations and combined to form a damage distribution using the analytic techniques first described in [1]. The calculations show that the high energy recoils from the nuclear inelastic reactions (calculated using the Monte Carlo code MCNPX [2]) produce a pronounced skewing of the damage energy distribution. While the nuclear elastic component (also calculated using the MCNPX) contributes only a small fraction of the total nonionizing damage energy, its inclusion in the shape of the damage across the array is significant. The Coulombic contribution was calculated using MRED [3-5], a Geant4 [4,6] application. The comparison with the dark current distribution strongly suggests that mechanisms which are not linearly correlated with nonionizing damage produced according to collision kinematics are responsible for the observed dark current increases. This has important implications for the process of predicting the on-orbit dark current response of the HgCdTe sensor array.
\end{abstract}

$\mathrm{M}$

\section{INTRODUCTION}

ANY emerging space astronomy programs will perform their science using infrared detectors in order to study the early Universe as well as Earth and planetary sciences, and the infrared bands are also important in other civil and military applications. Although we have observed hot pixel formation in proton-irradiated Rockwell IR hybrid detectors to be used in the James Webb Space Telescope (JWST) [8], we do not as yet understand the mechanism producing the hot pixels in

Manuscript received June 20,2006. The authors appreciate funding from the NASA Electronics and Packaging Program (NEPP), the DTRA Radiation Hardened Microelectronics Program and the James Web Space Telescope project. The authors also appreciate useful discussions with John Hubbs from Ball Aerospace.

C. J. Marshall and R. Ladbury are with the NASA-GSFC, Greenbelt, MD 20771 USA (corresponding author phone: 434-376-3402; fax: 703-991-6115; e-mail: cmarshal12@aol.com).

P. W. Marshall is a consultant, Brookneal, VA 24528 USA.

R. A. Reed, C. Howe, B. Weller, and M. Mendenhall are with Vanderbilt University, Nashville, TN 37235 USA.

A. Waczynski is with Global Science and Technology, Greenbelt, MD 20771 USA.

T.M. Jordan is with EMPC, Gaithersburg, MD 20885 USA.
HgCdTe. As a result we are unable to predict hot pixel formation on orbit. The purpose of this paper is to predict the proton-induced displacement damage distributions in $\mathrm{Hg}_{0.7} \mathrm{Cd}_{0.3} \mathrm{Te}$ detector arrays based on collision kinematics in order to see if they predict the observed dark current distribution.

In the case of Si sensors (including charge couple devices, active pixel sensors and charge injection devices) measurements show that the dark current distributions are often well explained by the damage distributions calculated based on collision kinematics [1,9-12]. Damage distributions were first calculated analytically by Marshall et al. in 1990 with good agreement obtained for dark current distributions produced by $12 \mathrm{MeV}$ protons in Si charge injection devices. At $63 \mathrm{MeV}$ the data indicated less variance in the measured distribution than in the damage energy calculation, a result also found by Hopkinson et al. at $100 \mathrm{MeV}$ in Si charge coupled devices (CCDs). Using the Monte Carlo code CUPID [13], Dale et al. showed that this result followed because the recoil ranges were comparable to the size of the dark current sensitive volume. In the limit of bulk material, both the analytic and the CUPID Monte Carlo approaches are in good agreement. As sensitive volumes shrink and incident proton energies increase, the ranges of the spallation recoil fragments approach the smallest dimension of the microvolume, and the pixel-to-pixel damage variance are best calculated using methods which track the damage deposition along the recoil atom pathlengths. In this regime, a Monte Carlo approach is well suited to describe the damage energy distribution.

Nevertheless, in some cases the Si dark current distributions cannot be described using collision kinematics alone. This has been attributed to hot pixel formation from electric field enhanced emission [e.g. 11, 14-17]. It is important to distinguish the two scenarios because in order to predict the hot pixel populations and assess dark signal nonuniformity, one needs to understand whether the underlying mechanisms are due to extraordinarily large damage regions from inelastic reactions in the pixel or by more ordinary damage in the presence of electric field enhanced emission which appears to follow from small damage regions in very small microvolumes filling only a tiny fraction of the pixel's volume. In the later case the hot pixel population may be expected to follow the Coulomb cross section $[14,17]$. 


\section{THE EXPERIMENT}

We use a previously measured dark current distribution of a Rockwell H2RG which is a hybrid device with a $2 \mathrm{k} \times 2 \mathrm{k}$ format and 18 micron pixel pitch. It incorporates a software configurable silicon readout circuit bump bonded to a $\mathrm{HgCdTe}$ detector array optimized for the JWST NIR $(0.6-5.5 \mu \mathrm{m})$ spectral range. Details of the experiment can be found in [8] but key details are provided here.

An engineering grade device was employed and displayed a number of cosmetic defects and 'hot' pixels that did not meet the stringent JWST flight focal plane array (FPA) operability requirements going into our test. A subset of $\sim 266,000$ pixels were extracted that were deemed to be "good" pixels as will be described in the next paragraph. The detector was held at 37 $\mathrm{K}$ and irradiated with $63 \mathrm{MeV}$ protons to a $5 \mathrm{krad}(\mathrm{Si})$ level which corresponds to a fluence of $3.7 \times 10^{10} \mathrm{~cm}^{-2}$. The dewar was maintained at temperature while being transported from the UC Davis Crocker Nuclear Laboratory back to the NASA Ames laboratory, and measurements were taken after residual radioactivity from the proton exposure had mostly decayed. Residual activity and cosmic ray effects were filtered out as described in [8].

As noted in [8], because of the very low dark current levels in these devices, the number of 'good' pixels was difficult to precisely quantify in the presence of read noise, cosmic-ray events and induced radioactivity in the cryostat. In order to isolate and remove engineering defects from the test data, a long series of darks was obtained in a clear environment with each device prior to the proton exposure. The distribution of dark currents estimated from the longest integration for each sensor chip assembly (SCA) was examined to determine the width of the peak in the histogram and thus the accuracy to which we could estimate dark current in these devices. This distribution width arises from a combination of system read noise and the true non-uniformity in dark current among the best pixels in the device. From the observed distributions it appeared that read noise was the greatest contributor to this width for each test SCA. We chose to set a dark current threshold for each long-integration frame in the dark current series at the median-pixel dark current plus the distribution width of the longest integration frame, as given by its fullwidth-half-maximum. By applying this threshold in each of the long-integration frames and removing from our select population any pixels that exceed the threshold in any of the frames, we derived a subpopulation of pixels that never exceed the dark current threshold over many hours of data collection. Post-irradiation data reduction is limited to this very conservative subpopulation of selected pixels. Note that this method is guaranteed to misidentify a number of good pixels as bad, since it makes no attempt to correct for the effects of cosmic ray events that occur during the long integration series, and because read noise will occasionally push marginally good pixels above the threshold. We consider this is not a problem in this work as we still had a sufficient population of pixels remaining to study the dark current distributions.

\section{DAMAGE ENERGy Distribution CalCUlations}

In this section we follow the method described in [1] for $\mathrm{Si}$, but with modifications, to calculate the damage energy distribution for $63 \mathrm{MeV}$ protons on $\mathrm{Hg}_{0.7} \mathrm{Cd}_{0,3} \mathrm{Te}$. The first step in the calculation of damage energy distributions is to calculate the interaction cross sections $(\sigma)$, as well as the mean damage energy, $(\mu)$ and the associated variance, $\left(\sigma^{2}\right)$ of the $\mathrm{Hg}_{0.7} \mathrm{Cd}_{0.3} \mathrm{Te}$ damage energy distributions due to Coulombic, nuclear elastic and nuclear inelastic interactions respectively. These means and variances correspond to the probability density function (pdf) governing the likelihood of a particular recoil energy resulting from a given proton- $\mathrm{Hg}_{0.7} \mathrm{Cd}_{0.3} \mathrm{Te}$ interaction.

Underlying assumptions include the fact that interaction mechanisms are random in nature, and for our purpose of examining the possible correlation of device dark current with displacement damage, we consider all non-ionizing energy; as with the related NonIonizing Energy Loss (NIEL) energy deposition rate. To achieve this, we use the Lindhard partition [18] as applied to the calculated recoil energy for a given ion and energy. We considered that the nonionizing energy associated with a recoil atom was deposited in the pixel in which it originated. In the next section we describe how the damage energy contributions from Coulomb scattering, nuclear elastic scattering, and nuclear inelastic scattering are determined, and show that each mechanism has its own probability density function (pdf) describing the probability of an individual pixel receiving a given amount of damage. As the three mechanisms are independent, we then show how relatively straight forward statistical tools can combine these pdfs in order to describe the damage distribution throughout the array; taking into account the pixel geometry, material composition, proton energy and proton fluence. Our treatment exercises these tools for the case of comparing a measured dark current distribution with a predicted damage energy distribution to test the hypothesis that there may be a linear correlation.

\section{A. Material and Recoil Spectrum Parameters}

The $\mathrm{Hg}_{0.7} \mathrm{Cd}_{0.3} \mathrm{Te}$ has a density of $7.41 \mathrm{~g} / \mathrm{cm}^{3}$ and a gram molecular weight of $151 \mathrm{~g}$. The JWST H2RG pixel area is 18 $\mu \mathrm{m}$ by $18 \mu \mathrm{m}$ and the HgCdTe layer is $\sim 10 \mu \mathrm{m}$ thick. From measurements on other $\mathrm{HgCdTe}$ detectors we expect the diffusion length to be at least $10 \mu \mathrm{m}$, so dark current should be collected from the entire pixel volume, even though the diode junction occupies a small fraction $(-10 \%)$ of the pixel volume. In this geometry, the volume and density are large enough so that we do not expect the ranges of the recoils to be long compared to the volume dimensions so the analytic approach should offer a valid approximation.

The results for the recoil spectrum parameters are shown in Table 1, along with the MRED (Monte Carlo Radiative Energy Deposition) [3-5] results for the Coulomb parameters in the units indicated. MRED is a Geant 4 based tool that employs a shielded Coulomb potential. In the future, we plan to develop MRED for a more comprehensive and general solution but for now we are using it for a "point" solution for the case of $3.7 \mathrm{x}$ $10^{10} \mathrm{~cm}^{-2} 63 \mathrm{MeV}$ protons on the pixel geometry cited (i.e. 1.2

To be published in Transactions on Nuclear Science (TNS) Special Edition, Institute of Electrical and Electronics Engineers (IEEE), June 2007. 
X $10^{5}$ protons/pixel). This proton exposure results in 5 $\operatorname{krad}(\mathrm{Si})$.

TABLE 1

Proton Nuclear Reactions on $\mathrm{HgCdTe} x=0.3$

\begin{tabular}{|c|c|c|c|c|}
\hline $\begin{array}{l}\text { Proton } \\
\text { Energy } \\
\text { (MeV) }\end{array}$ & $\begin{array}{l}\text { Cross } \\
\text { Section } \\
\text { (barns) }\end{array}$ & $\begin{array}{l}\text { Mean } \\
\text { Recoil } \\
\text { Energy } \\
(\mathrm{MeV})\end{array}$ & $\begin{array}{c}\text { Mean } \\
\text { Damage } \\
\text { Energy } \\
\text { (MeV) }\end{array}$ & $\begin{array}{c}\text { Variance of } \\
\text { Damage } \\
\text { Energy } \\
(\mathrm{MeV})^{2}\end{array}$ \\
\hline \multicolumn{5}{|c|}{ Nuclear Elastic Reactions } \\
\hline 63 & 1.566 & 0.0367 & 0.0236 & $5.56 \times 10^{-3}$ \\
\hline \multicolumn{5}{|c|}{ Nuclear Inelastic Reactions } \\
\hline 63 & 1.599 & 0.365 & 0.204 & $2.87 \times 10^{-2}$ \\
\hline \multicolumn{5}{|c|}{ Coulombic Interactions } \\
\hline 63 & & & $0.734^{*}$ & $9.60 \times 10^{-13_{* *}}$ \\
\hline
\end{tabular}

$* \mathrm{MeV}$ per pixel with incident $63 \mathrm{MeV}$ proton fluence of $3.7 \times 10^{10} \mathrm{~cm}^{-2}$ ** $\mathrm{MeV}^{2}$ per pixel with a $63 \mathrm{MeV}$ proton fluence of $3.7 \times 10^{10} \mathrm{~cm}^{-2}$.

We arrived at the distribution describing Coulomb damage based on the mean damage energy deposited in a large number of Monte Carlo runs (using MRED [3-5]]) for the pixel geometry and proton fluence of $3.7 \times 10^{10} \mathrm{~cm}^{-2} 63 \mathrm{MeV}$ protons. Using 100 separate simulations of the mean damage, we calculated the sample mean presented in the Table. It is interesting to note that the mean damage energy per pixel calculated using the NIEL value in Fig. 1 (based on the analytic Ziegler Biersack Littmark (ZBL) method [20]) agrees to within $17 \%$ with the value obtained using the MRED Monte Carlo runs.

Calculation of the sample variance for the 100 simulations yields the surprisingly small value listed in Table 1 . Note that each simulation incorporated a new random number seed, and each case was run for exactly $1.20 \times 10^{5}$ protons/pixel. Consideration of our test condition and inclusion of the variance associated with the Poisson probability describing the incident particle fluence associated with an average of $1.2 \mathrm{x}$ $10^{5}$ protons/pixel yields a much larger variance of $4.49 \times 10^{-6}$ $\mathrm{MeV}^{2}$. The variance used in the following calculations is therefore very heavily dominated by this Poisson contribution, and for such a large incident fluence we have confidence in assuming a Gaussian form for the distribution of Coulomb damage throughout the array. Inspection of the variance indicates very little difference in the Coulomb damage from pixel-to-pixel. This follows from the fact that the mean energy imparted per Coulomb scattering event is on the order of the displacement threshold $(\sim 20 \mathrm{eV})$, and this results in the generation of isolated Frenkel pairs (and possibly point defects in $\mathrm{HgCdTe}$ ). So the Coulomb damage is spread very evenly throughout a pixel and hence the pixel-to-pixel uniformity is expected. This parallels the treatment of proton damage from Coulomb scattering in $\mathrm{Si}$ in $[19,20$ and references therein].

The nuclear elastic component (calculated using the Monte Carlo code MCNPX [2]) has an almost negligible effect on the
NIEL (Fig. 1) as it is typically an order of magnitude below the dominant term across the entire energy range. But as we will demonstrate later, consideration of the nuclear elastic contributions has important consequences for the shape of the composite damage distribution. We can see from the table that the mean recoil energy and variance are an order of magnitude below that of the inelastic interactions, but their cross sections are essentially equal. The difference in damage energies per event gives rise to the lower NIEL relative to the nuclear inelastic events, and this is also illustrated in Fig. 2 which shows the comparison of damage energy distributions for nuclear events generated in the MCNPX calculations.

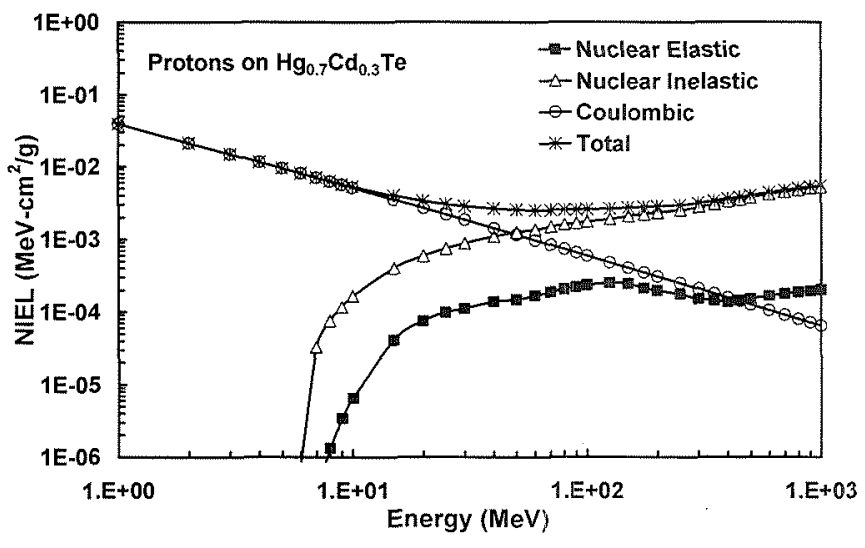

Fig. 1 Proton NIEL in $\mathrm{Hg}_{0.7} \mathrm{Cd}_{0.3} \mathrm{Te}$ as calculated in [21]. Note that the NIEL is very insensitive to the exact stoichiometry.

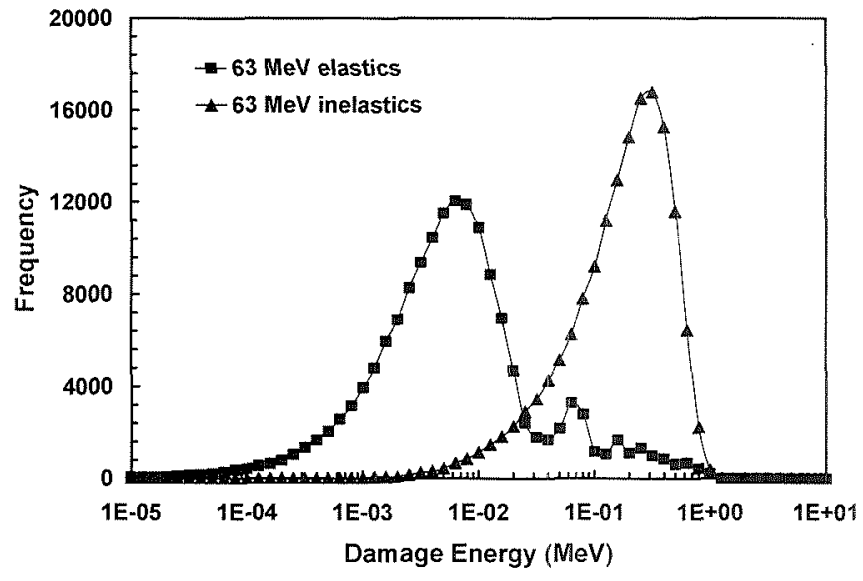

Fig. 2 Comparison of the damage energy distributions for the nuclear elastic and nuclear inelastic interactions at $63 \mathrm{MeV}$. Note the abscissa is on a $\log$ scale to represent the two distributions on the same figure.

The method used to compute the nuclear contribution to NIEL and the associated variance is based on the thin target approximation using MCNPX and a methodology developed by Jun [22]. A thin cylindrical disc of the material of interest with a normalized density of 0.01 atoms/barn-cm was modeled, and a simulated pencil beam of protons penetrates the material. Using the damage energy tally, the history tape written by MCNPX was analyzed to calculate the mean

To be published in Transactions on Nuclear Science (TNS) Special Edition, Institute of Electrical and Electronics Engineers (IEEE), June 2007. 
damage energy per source particle, $T_{\text {dam }}$, which is the nonionzing portion of energy deposited (i.e. after application of the Lindhard partition function). Then, NIEL is calculated by:

$$
S_{N I E L}=\left(\frac{N_{A}}{A}\right) \frac{T_{d a m}}{\left(N_{v} \cdot x\right)}
$$

where $N_{A}$ is Avogadro's number, $A$ is the gram atomic weight of the target material, $N_{v}$ is the atom density and $x$ is the target thickness. By using MCNPX, we were able to compute the nuclear contributions to the proton NIEL for each material and then superimpose them to arrive at the NIEL for the $\mathrm{Hg}_{0.7} \mathrm{Cd}_{0.3} \mathrm{Te}$. The production of displaced atoms is dominated by the Coulombic interactions below $10 \mathrm{MeV}$, while the nuclear collisions (particularly the nuclear inelastic) take over at energies exceeding $\sim 50 \mathrm{MeV}$. For the Coulombic NIEL shown in Fig. 1, the calculation was done analytically using the ZBL method. This analytic approach provides the mean, but as previously mentioned the MRED calculations were necessary to determine the variance for Coulomb scattering events. Mean values and their variances for nuclear elastic and inelastic damage distributions were determined using the history tape out of MCNPX for the distributions shown in Fig. 2, and later we refer to these as the moments of the single event probability density functions for describing nuclear collision damage.

\section{B. Calculated Damage Distributions}

The cross sections for nuclear scattering events to produce damage listed in Table 1, combined with the proton fluence chosen to represent a relevant JWST mission exposure, result in only a few events in the H2RG pixel volume. We define $\eta$ as the average number of recoils per pixel and it is given by

$$
\eta=\sigma \cdot \Phi \cdot \rho \cdot V_{\text {pixel }} \cdot N_{A} / A
$$

where $\sigma$ is the interaction cross section, $\Phi$ is the proton fluence, $\rho$ is the $\mathrm{HgCdTe}$ density, $V$ is the pixel volume, $N_{A}$ is Avogadro's number and $A$ is the gram molecular weight of $\mathrm{Hg}_{0.7} \mathrm{Cd}_{0.3} \mathrm{Te}$. We find that the average number of nuclear elastic and inelastic recoils per pixel is 5.7 and 5.8 respectively, and the maximum number of events expected for any pixel is $\sim 20$ for our pixel population. Also note that only $\sim 800$ pixels out of $\sim 266,000$ have no nuclear elastic interactions, and similarly $\sim 800$ pixels have no inelastic collisions. As these are independent variables, the population of pixels having only Coulomb damage is given by the product of the Poisson probability of no nuclear elastics and the probability of no nuclear inelastics which yields only $\sim 2$ pixels! From this we see that even though the total damage from nuclear elastic processes in small, the consideration of this damage is very important in arriving at the appropriate distribution describing pixels with no nuclear inelastic collisions, essentially adding to the tail of the Coulombic contribution thereby increasing the variance. Obviously where nuclear inelastic collisions are present their importance in the damage of a given pixel plays a dominant role.

Since the damage from Coulombic, nuclear elastic and nuclear inelastic events are each independent and random variables, the statistical description of the cumulative damage can be approached by evaluating them independently and then combining by convolution. The functional form of the Coulomb portion is Gaussian arising from the fact that each pixel has a very large number of relatively small collisions. Inspection of the functional form of the damage distributions of Fig. 2 reveals an asymmetric distribution which is skewed towards higher energies (note the abscissa is on a log scale to represent the two distributions on the same figure). After considering several candidate distributions, we determined that the two parameter gamma function is well suited for describing both the nuclear elastic and nuclear inelastic damage functions (as in [1]). The gamma function is expressed as $\Gamma\left(\mu^{*} \lambda\right)$ where $\mu$ is the mean recoil energy and $\lambda=\mu / \sigma^{2}$ where $\sigma^{2}$ is the associated variance. This result affords a very convenient approach for the techniques we use to combine distributions in that the convolution of two gamma functions yields a new gamma function. This fact, along with the result that distribution means and variances add linearly under convolution, affords a concise approach to this analytic technique. In addition, since the Gaussian function is a special case of the gamma function, their combination under convolution results in a gamma function and this result means we have a simplified path to combining all three independent variables without requiring FFT-based convolution methods.

Using the technique just described, we proceed to combine Coulombic damage with the single event probability function (SEpdf) for elastic nuclear reactions. This step reduces to defining a new probability density function based on the gamma function (with added means and variances) as shown in equation below. The probability $(\mathrm{P})$ of having the damage energy $(\mathrm{DE})$ is

$$
P(D E)=\frac{\lambda}{(\lambda \cdot D E)^{1-r}} \cdot \frac{e^{(\lambda \cdot D E)}}{\Gamma(r)}
$$

where and $\lambda=\mu / \sigma^{2}$ and $r=\mu^{2} / \sigma^{2}$. (This function is also normalized to unit area.) For convenience, we note that a gamma function is defined as

$$
\Gamma(\alpha)=\int_{0}^{\infty} x^{(\alpha-1)} \cdot e^{-x} d x
$$

where $\alpha>0$ and $\mathrm{x}$ is the independent variable. For example, the damage probability function (Equation 3) applies to pixels that have Coulombic damage and only 1 nuclear elastic recoil for which $\mu$ is the sum of the Coulombic mean damage energy and the mean damage energy for one elastic recoil, and likewise, $\sigma^{2}$ is the sum of the Coulomb variance and the variance for one nuclear elastic recoil. By extension we generate a family of gamma distributions accounting for pixels with multiple nuclear elastic recoils by examining the $\mathrm{N}$-fold convolution of the SEpdf with itself to get the pdf for a pixel with $\mathrm{N}$ elastic recoils. This is done for $\mathrm{N}=2,3,4$, etc, up to

To be published in Transactions on Nuclear Science (TNS) Special Edition, Institute of Electrical and Electronics Engineers

(IEEE), June 2007. 
the maximum $\mathrm{N}=19$ events expected to occur for the given population of pixels. The resulting total elastic (combined Coulomb plus nuclear elastic) pdfs for a pixel with $\mathrm{N}$ nuclear elastic recoils is shown in Fig. 3. Note that as $\mathrm{N}$ increases the mean of the distribution increases, it broadens and also tends towards a gaussian - all expected based on the Central Limit Theorem.

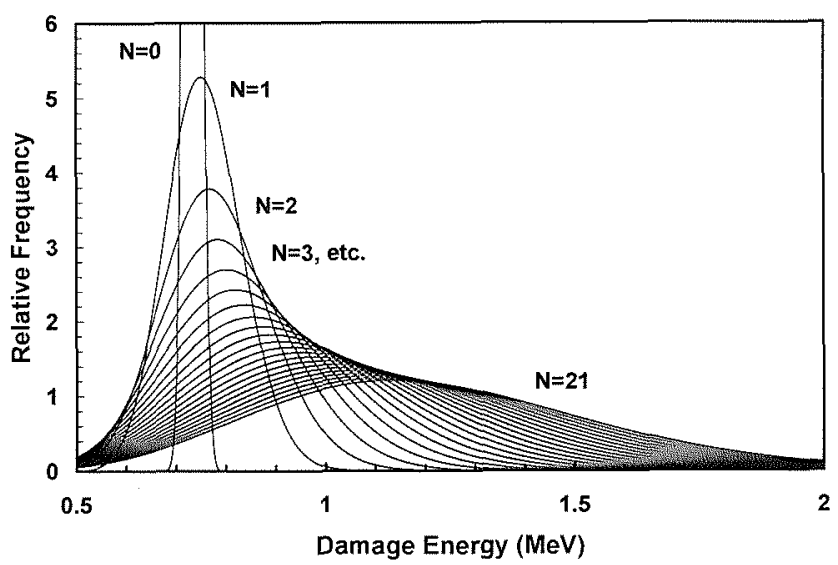

Fig. 3 The total elastic pdfs for a pixel with $N=0,1,2, \ldots 21$ nuclear elastic interactions. (The $\mathrm{N}=0$ (Coulombic interactions only) is a gaussian curve with a maximum value of 29.5 .)

For the sensitive volumes $\left(\mathrm{V}_{\text {pixel }}\right)$ and fluence $(\Phi)$ of interest the variation in the number of nuclear elastic (or inelastic) recoils per pixel is described by a discrete Poisson distribution in which the probability $P$ is

$$
P(x, \eta)=\frac{\left(e^{-\eta} \cdot \eta^{x}\right)}{x !}
$$

where $\mathrm{x}=0,1,2, \ldots$ recoils per pixel and $\eta$ is the average number determined according to Equation 2. Using this relation, we weight each of the 19 gamma distributions with the Poisson probability for $\mathrm{x}$ (nuclear elastic) recoils in a pixel, and then sum them to arrive at the combined Coulomb and nuclear elastic (which we will refer to as the combined elastic) damage energy distribution across the array. Note that $\sum \mathrm{P}(\mathrm{x}, \eta)$ $=1$.

In Fig. 4 we present the Poisson weighted pdfs as well as the sum in graphical form, and using standard statistical techniques we calculate the mean and variance of this resulting combined elastic function to be $0.87 \mathrm{MeV}$ and $0.19 \mathrm{MeV}^{2}$. Note that the Poisson weighting and superposition results in a functional form which is not described exactly by a gamma function, but due to the ease of working with gamma distributions for the next step of the analysis to incorporate nuclear inelastic damage, we approximate the resulting combined elastic distribution with a gamma function having the same mean and variance. This function is also shown in Fig. 4. The comparison shows a reasonably good fit, and we consider the tradeoffs associated with this assumption to favor this approximation.
Note that the variance in the combined elastic damage is four orders of magnitude greater than the Poisson dominated Coulomb only case. Even though nuclear elastic collisions impart only a small fraction of the total NIEL, their inclusion in the population of pixels in the array ( $\sim 800$ total) which have no inelastic damage is necessary.

Fig. 1 and Table 1 reveal the important implications of the nuclear inelastic damage in that it accounts for $>60 \%$ of the total damage at $63 \mathrm{MeV}$, and with an average of 5.8 events per pixel the variation from pixel-to-pixel is obviously the most significant aspect of the distribution of damage throughout the array. Using the techniques already described, we combine the $\mathrm{N}$-fold convolution of up to 20 inelastic events per pixel with the gamma function describing the combined elastic damage and apply Poisson weighting per Equation 4 and finally perform the summation of all the Poisson weighted distributions.

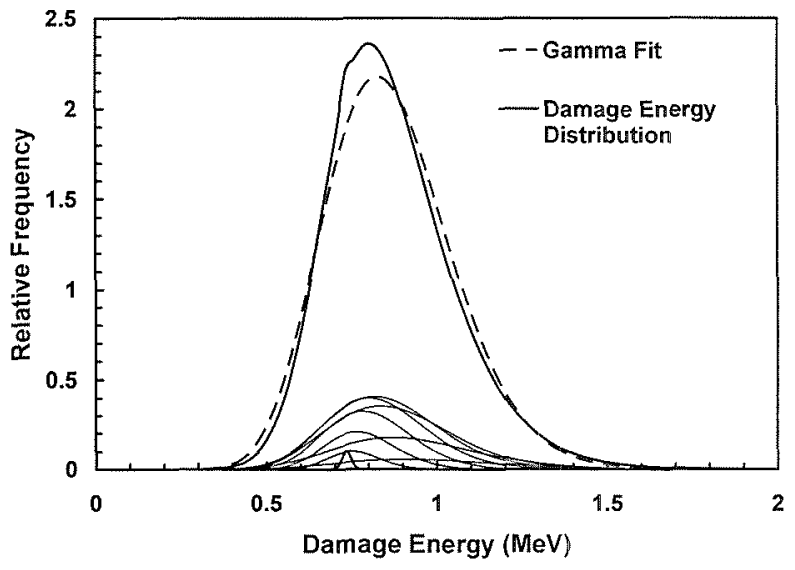

Fig. 4 Comparison of the combined elastic damage energy distribution and a gamma function with the same mean and variance. The distributions underneath the two curves are the Poisson weighted gamma distributions from Fig. 3 for pixels with $\mathrm{N}$ nuclear elastic recoils which are summed to arrive at the total elastic damage energy distribution. For clarity only $\mathrm{N}=0-6,8,10$ are shown. Note the reduced variance for the $\mathrm{N}=0$ (Coulomb only) case. Recall that the average number of nuclear elastics is 5.7 .

The calculated damage distribution is shown in Fig. 5, which shows the distribution of pixels with elastic damage plus $0,1,2$, etc. inelastic interactions underneath the sum of each contribution. The average number of inelastic collisions per pixel was $\sim 5$, and the maximum number of inelastic recoils expected per pixels is 20 for our fluence and pixel population. As one can see, skewness in the distribution is primarily attributed to the Poisson weighting of the inelastic interactions. This function describes the distribution of damage corresponding to our proton-induced dark current distribution. Note that the shape assumed for the SEpdfs (gamma functions in our case) is not very critical.

It is interesting to compare the damage distribution calculated for a high atomic number compound such as $\mathrm{HgCdTe}$ with the previous Si result [1]. In HgCdTe the effect of the inelastics is much more prominent - a higher mean energy and a broader peak with a more pronounced tail is 
observed because the inelastic cross section is 3 times larger, the mean recoil damage energy is $30 \%$ higher and the variance is an order of magnitude larger in $\mathrm{HgCdTe}$ as compared to $\mathrm{Si}$.

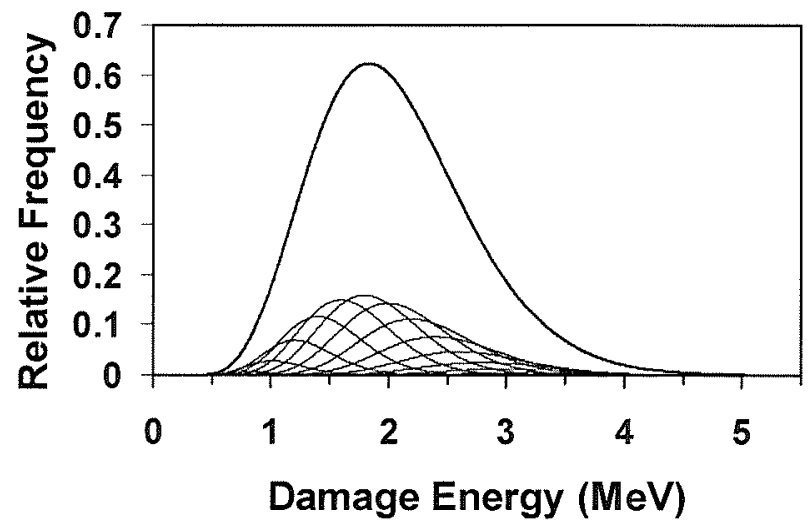

Fig. 5 Calculated damage energy distribution for 266,000 $\mathrm{Hg}_{0.7} \mathrm{Cd}_{0.3}$ Te pixels irradiated with $3.7 \times 10^{10} \mathrm{~cm}^{-2} 63 \mathrm{MeV}$ protons. Below the summed damage distribution we show the Poisson weighted damage distributions for the pixels with only elastic events (left most peak barely visible in figure), and the pixels with elastic events plus 1 (left peak with maximum at $\sim 1 \mathrm{MeV}$ ), 2,3...18 inelastic interactions per pixel. (Note that a pixel may contain up to 18 inelastic interactions though only distributions with up to 10 are resolved in the plot.)

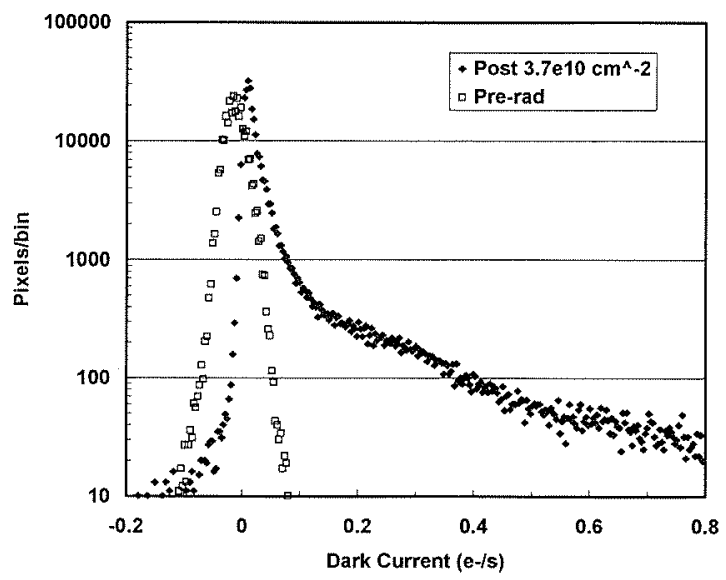

Fig. 6 Measured dark current distribution for $266,000 \mathrm{Hg}_{0.7} \mathrm{Cd}_{0.3}$ Te pixels before irradiation and after irradiation with $3.7 \times 10^{10} \mathrm{~cm}^{-2} 63 \mathrm{MeV}$ protons. The data represent measured absolute values and the negative numbers reflect measurement noise in the system. The mean dark current is extremely small.

\section{COMPARISON OF DAMAGe Distribution WITH DARK CURRENT HISTOGRAM}

The measured dark current histogram for 266,000 selected pixels is shown in Fig. 6 along with the pre-irradiation histogram. In [8], the slight median shift was not investigated, and hence was not presented as necessarily real. However, we have re-analyzed this data and do find a small shift in the median dark current after irradiation to $3.7 \times 10^{10} \mathrm{~cm}^{-2} 63$ $\mathrm{MeV}$ protons as indicated in the figure. It is apparent in Fig. 7 that the calculated damage distribution does not predict the measured dark current distribution which indicates that some other mechanism than collision kinematics is also responsible for the high dark current pixels seen in the data. This makes an on-orbit prediction of the dark current based on proton results at a single energy (63 MeV in our case) problematic since the nonionizing energy loss rate (NIEL) correlation does not appear to hold. Measurements at $8 \mathrm{MeV}$ are planned to see if the high dark current pixels correlate with the Coulombic portion of the NIEL which would be the case if electric field enhanced emission via trap-assisted tunneling is responsible as has been seen in Si. (Trap-assisted tunneling is known to be important in $\mathrm{HgCdTe}$ sensors at low temperatures.) If this is also the case with the present $\mathrm{HgCdTe}$ array then first order estimates of hot pixel rates expected on-orbit which are based on dark current histograms for the $63 \mathrm{MeV}$ equivalent fluence for a given mission will underestimate the number of hot pixels.

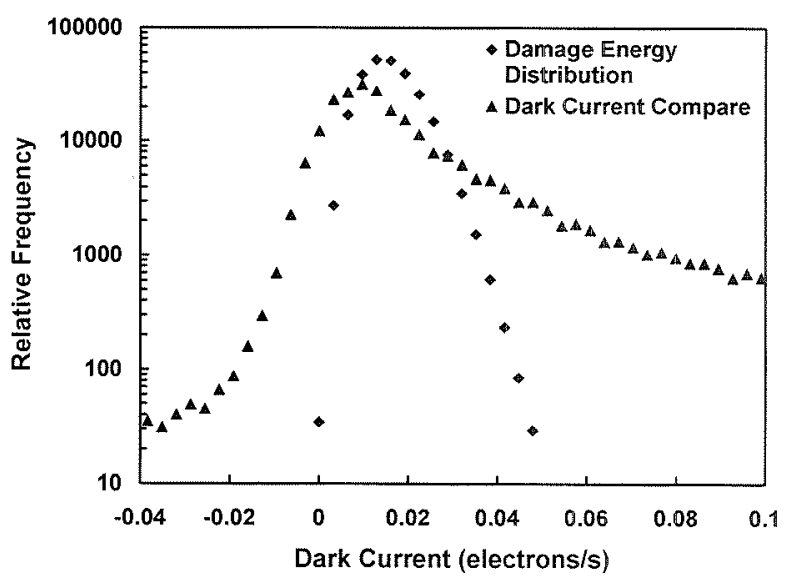

Fig. 7 Comparison of measured dark current histogram with damage energy calculation. The high dark current pixels are not the result of collision kinematics.

\section{DISCUSSION AND SUMMARY}

In comparison to earlier analytic damage energy distribution predictions (e.g. [1]) we find that the role of the inelastics in producing the highly damaged pixels is even more dominant for the $\mathrm{HgCdTe}$ case as compared to $\mathrm{Si}$. The cross section for inelastic interactions is larger in the (higher atomic number system) $\mathrm{HgCdTe}$ by a factor of three and the variance is an order of magnitude larger in $\mathrm{HgCdTe}$.

We have developed a new combined Monte Carlo and analytic approach to the calculation of the pixel-to-pixel distribution of proton-induced damage in a $\mathrm{HgCdTe}$ sensor array and compared the results to measured dark current distributions after damage by $63 \mathrm{MeV}$ protons. The moments of the Coulombic, nuclear elastic and nuclear inelastic damage distributions were extracted from Monte Carlo simulations and 
used to generate single event probability distributions describing each class of proton- $\mathrm{HgCdTe}$ interaction. These were combined as discussed in the text to form a damage energy distribution. The calculations show that the high energy recoils from the nuclear inelastic reactions (calculated using the Monte Carlo code MCNPX [2]) produce a pronounced skewing of the damage energy distribution. While the nuclear elastic component (also calculated using the MCNPX) contributes only a small fraction of the total nonionizing damage energy, its inclusion in the shape of the damage across the array is significant because its variance dominates in those pixels with no inelastic reactions. The Coulombic contribution was calculated using MRED [3-5], a Geant4 $[4,6]$ application. We noted that only 2 pixels in the entire array have only Coulombic interactions. The comparison with the $63 \mathrm{MeV}$ proton-induced dark current distribution strongly suggests that mechanisms which are not linearly correlated with nonionizing damage produced according to collision kinematics are responsible for the high dark current pixels. Measurements at $8 \mathrm{MeV}$ are planned to see if the high dark current pixels correspond to the Coulombic portion of the NIEL which would be the case if electric field enhanced emission is responsible as has been seen in Si. The technique for describing the damage energy distributions is extendable to other proton energies, material systems, and pixel geometries.

\section{REFERENCES}

[1] P. W. Marshall, C. J. Dale and E. A. Burke, "Proton-induced displacement damage distributions and extremes in silicon microvolumes," IEEE Trans. on Nucl. Sci., Vol. 37, pp. 17761783, December 1990.

[2] "MCNPX User's Manual: Version 2.4.0," LA-CP-02-408, Los Alamos National Lab, September 2002.

[3] H. Mendenhall and R.A. Weller, "An algorithm for computing screened Coulomb scattering in Geant4," Nuclear Instruments and Methods A Vol. 227, pp. 420-430, 2005.

[4] R.A. Weller, M.H. Mendenhall, and D.M. Fleetwood, "A screened Coulomb scattering module for displacement damage computations in Geant4," IEEE Trans. Nucl. Sci, Vol.. 51, pp. 3669-3678, 2004.

[5] M. H. Mendenhall and R. A. Weller, "Algorithms for the rapid computation of classical cross sections for screened Coulomb collisions," NIM B58, pp. 11-17 (1991).

[6] S. Agostinelli et al., "Geant4 -A simulation toolkit," Nucl. Instrum. Meth. Phys. Res., Vol. A 506, pp. 250-303, 2003.

[7] Geant4 [online]. Available: http://geant4.web.cern.ch/geant4

[8] M.E. McKelvey, K.A. Ennico, R.R. Johnson, P.W. Marshall, R.E. McMurray Jr., C.R. McCreight, J.C. Pickel and R.A. Reed, "Radiation environment performance of JWST prototype FPAs," SPIE Proceedings, Focal Plane Arrays for Space Telescopes, Vol. 5167, pp.223-234, 2003.

[9] G.R. Hopkinson, "Cobalt-60 and Proton Radiation Effects on Large Format, 2-D, CCD Arrays for an Earth Imaging Application," IEEE Trans. Nucl. Sci., Vol. 39, No. 6, pp. 20182025, 1992.

[10] M.S. Robbins, "High-Energy Proton-Induced Dark Signal in Silicon Charge Coupled Devices," IEEE Trans. Nucl. Sci., Vol. 47, No. 6, pp. 2473-2479, 2000.
[11] J. Bogearts, B. Dierickx, and R. Mertens, "Enhanced Dark Current Generation in Proton-Irradiated CMOS Active Pixel Sensors, Trans. Nucl. Sci., Vol. 49, No. 3, pp. 1513-1521, 2002.

[12] R. Germanicus, S. Barde, L. Dusseau, G. Rolland, C. Barillot, F. Saigne, R. Ecoffet, P. Calvel, J. Fequet, and J. Gasiot, "Evaluation and Prediction of the Degradation of COTS CCD Induced by Displacement Damage," IEEE Trans. Nucl. Sci., Vol. 49, No. 6, pp. 2830-2835, 2002.

[13] C.J. Dale, L. Chen, P.J. McNulty, P.W. Marshall, and E.A. Burke, "A comparison of Monte Carlo and analytic treatments of displacement damage in Si microvolumes," IEEE Trans. Nucl. Sci., Vol. 41, No. 6, pp. 1974-1983, 1994.

[14] P.W. Marshall, C.J. Dale, E.A. Burke, G. P. Summers, and G. E. Bender, "Displacement Damage Extremes in Silicon Depletion Regions," IEEE Trans. Nucl. Sci., Vol. 36, No. 6, pp. 1831$1839,1989$.

[15] J.R. Srour and R.A. Hartman, "Enhanced Displacement Damage Effectiveness in Irradiated Silicon Devices," IEEE Trans. Nucl. Sci., Vol. 36, No. 6, pp. 1825-1830, 1989

[16] I.H. Hopkins and G.R. Hopkinson, "Further Measurements of Random Telegraph Signals in Proton-Irradiated CCDs," IEEE Trans. Nucl. Sci., Vol. 42, No. 6, pp. 2074-2081, 1995.

[17] C.J. Marshall, P.W. Marshall, A. Waczynski, E.J. Polidan, S.D. Johnson, R.A. Kimble, R.A. Reed, G. Delo, D. Schlossberg, A.M. Russell, T. Beck, Y. Wen, J. Yagelowich, R.J. Hill, "Hot pixel annealing behavior in CCDs irradiated at $-84^{\circ} \mathrm{C}$," IEEE Trans. Nucl. Sci., Vol. 52, No. 6, pp. 2672-2677, 2005.

[18] J. Lindhard, V. Nielsen, M. Scharff and P.V. Thomsen, Mat. Fys. Medd. Dan. Vid. Selsk., Vol. 33, No. 10, 1963.

[19] C.J. Dale, P.W. Marshall, E.A. Burke, G.P. Summers, and E.A. Wolicki, "High Energy Electron Induced Displacement Damage in Silicon," IEEE Trans. Nucl. Sci., NS-35, pp. 1208-1214, 1988.

[20] J.R. Srour and J.W. Palko, "A framework for understanding displacement damage mechanisms in irradiated Silicon devices," IEEE Trans. Nucl. Sci., NS-53, pp. 3610-3620, 2006.

[21] B.C. Fodness, P.W. Marshall, R.A. Reed, T.M. Jordan, J.C. Pickel, I. Jun, M.A. Xapsos, E.A. Burke and R. Ladbury, "Monte Carlo Treatment of Displacement Damage in Bandgap Engineered HgCdTe Detectors," Proceedings of the $7^{\text {th }}$ European Conference on Radiation and its Effects on Components and Systems, pp. 479-485, 2003.

[22] I. Jun, M.A. Xapsos, S.R. Messenger, E.A. Burke, R.J. Walters, G.P. Summers and T.M. Jordan, "Proton NonIonizing Energy Loss (NIEL) for device applications," IEEE Trans. Nucl. Sci., Vol. 50, No. 6, pp. 1924-1928, 2003. 